\title{
Self-Regulation, Mind Wandering, and Cognitive Absorption During Technology Use
}

\author{
Yulia W. Sullivan \\ Baylor University \\ Yulia Sullivan@,baylor.edu
}

\author{
Fred Davis \\ Texas Tech University \\ Fred.Davis@,ttu.edu
}

\begin{abstract}
Interaction with technology involves not only externally directed cognition, but also internally directed cognition. Although the information systems (IS) field has made a significant progress toward understanding of how individuals use technology, more emphasis has been given to goal-directed external activity that requires focused external attention and less or no emphasis on goal-directed internal activity called mind wandering. Drawing upon the emerging cognitive neuroscience literature, the current research investigates the relationships between self-regulation, mind wandering, and cognitive absorption. Specifically, we hypothesize there is a U-shape relationship between mind wandering and cognitive absorption. Based on a cross-sectional study of 323 individuals, the results reveal that the relationship between mind wandering and cognitive absorption is curve-linear. As mind wandering increases, cognitive absorption decreases to a certain point, after which, cognitive absorption increases as mind wandering increases. The results also show self-regulation has a significant effect on mind wandering and cognitive absorption.
\end{abstract}

\section{Introduction}

One of the most developed streams of research in the information systems (IS) discipline is individuals' use of information technology across different adoption phases (i.e., from initial adoption to postadoption). In this line of research, the concept of goaldirected behavior has been the core element of theorizing IS use behaviors. In order to optimize the benefits of technology, users have to use it in a way that helps them attain the relevant goal and meet specific task demands [1]. Although the field has made a significant progress toward understanding of how users use technology, more emphasis has been given to goal-directed external activity or task-dependent activity that requires users' focused external attention. Less or no emphasis is given to internal cognition or goal-directed internal activity called self-generated thought or mind wandering-spontaneous, selfgenerated thoughts that arise from internal mental processes that are largely independent of an external input [2]. Unlike external cognition, internal cognition requires sustained internally directed attention and involves mind wandering [3]. Given many cognitive activities involved in the interaction between users and technology (e.g., innovation, idea generation, problem solving, etc.) require internally directed cognition, it is important for the IS discipline to include mind wandering as a subject of research. Furthermore, although mind wandering can lead to low task performance $[4,5]$, the functional benefits of mind wandering have been reported in a number of tasks, including planning, mental simulation, and creative thinking tasks [6]. These contradictory findings have led researchers to argue that mind wandering should be viewed as a complex form of cognition worthy of empirical investigation $[2,7]$.

Driven by the need to study goal-directed internal activity in IS research, in this paper, we focus on understanding the role of mind wandering in influencing technology use, measured as cognitive absorption. We argue that although mind wandering can have a negative effect on cognitive absorption, it can also have a positive effect when it reaches a point where users use the experience of mind wandering to cope with the task demands (i.e., a curve-linear relationship). Further, according to resource allocation theory, self-regulation is the driver force for goaldirected behaviors and is responsible for directing and maintaining attentional control [8]. The distribution of resources during a task execution is determined by self-regulatory activities [8]. Thus, we argue that selfregulation plays a significant role in the generation of mind wandering experience.

Drawing on the theory of mind wandering and selfregulatory theory, we address the following research questions: (1) what is the effect of self-regulation on mind wandering and absorptive capacity? and (2) will different levels of mind wandering influence absorptive capacity differently? Our study contributes to the literature in several ways. First, we establish our 
conceptual model based on the findings from prior neuroimaging studies by mapping the brain networks to their cognitive constructs discussed in our study. Second, we theorize a curve-linear relationship between mind wandering and cognitive absorption. Our study is one of the first studies that investigate mind wandering during technology use.

\section{Theoretical Background}

Due to the absence of theory describing the relationship between internal and external cognition, this section describes the need to look outside IS research to find and review the relevant literature associated with mind wandering from the reference disciplines. Based on our review, we then develop a theoretical model used as a foundation for our current research.

Mind wandering, also known as "self-generated thoughts" or "stimulus-independent thoughts" make up a significant portion of our daily lives [9, 10]. Mind wandering is defined as "a shift in attentional focus toward unrelated self-generated information at the cost of task-relevant perceptual stimuli" [11, p. 32]. A recent study reported that individuals spend between 30 to 50 percent of their waking hours engaged in mind wandering [10]. We might think about our summer vacation plans while working on a manuscript or think about a research project while driving. Mind wandering experience is distinct from other cognitive behaviors in that it is not derived directly from an external stimulus; rather it forms a train of internally generated thoughts, perceptually decoupled from external environments and tasks being performed [2, 12]. Findings from prior cognitive psychology and neuroscience studies suggest that mind wandering shares a number of common attributes with goaldirected thought, which occurs frequently during reasoning, problem solving, and decision-making tasks [13]. Further, neuroimaging evidence has demonstrated that the brain regions recruited during mind wandering overlap with those recruited during goal-directed thoughts [14]. Thus, despite our sense that mind wandering occurs at a significant cost to performance, it could potentially serve some functional benefits.

Understanding the psychological and neural mechanisms underlying mind wandering has been a major research topic in the cognitive and neuroscience disciplines in recent years [11]. Neuroimaging studies have revealed that mind wandering is linked to a brain network called the default mode network (DMN). The landmark discovery of the DMN has provided a viable starting point from which to understand the brain basis of mind wandering $[15,16]$. Early research has shown this network usually becomes active when individuals are in a resting state and not focused on the outside world and inactive during externally focused goaldirected tasks [9]. However, the most recent development of research in this area offers new insights into the functions of the DMN. According to this new perspective, the DMN serves important psychological functions [9] and its activity is attributed to internally focused thought that can occur in the form of mind wandering if it takes place simultaneously with, and yet is unrelated to an ongoing task [13, 17]. Thus, the link between the DMN and mind wandering may suggest that mind wandering is beneficial to certain types of goal-directed tasks [17].

In order to establish a theoretical foundation for our conceptual model, we reviewed the literature on the DMN and its relation to other brain networks published in the major journals from the reference disciplines. Despite some differences and inconsistency of findings among these prior studies, researchers are largely in agreement on supporting the link between the DMN and mind wandering. The literature suggests that the DMN increases its activity during goal-directed cognitive tasks, including autobiographical memory, self-reference, and creative thinking tasks. There are three most consistently engaged regions within the DMN: (1) the medial parietal cortex/posterior cingulate cortex (PCC); (2) the medial prefrontal cortex (MPFC); and (3) the medial temporal lobe (MTL).

The PCC can be viewed as an important region that contributes to self-related processing, social cognitive processing, disruptive attention [18], and bottom-up attention including managing behaviorally relevant source of information drawn from memory and/or perception [11]. The MPFC functions are associated with self-related processing, including when individuals retrieve personal knowledge, recall autobiographical memories, consider their future goals, and simulate personal future events [11]. Together, the PCC and MPFC comprise a "core", "mental", and "minimal" self [19] and an emergent outcome of the association between the PCC and MPFC is the mental construction of an overarching personal meaning which can guide thoughts and behaviors underlying mind wandering experience. Finally, the MTL subsystem serves a critical role in retrieving long-term declarative memory [11]. This region is consistently activated during goal-directed tasks, including selfprojection [20], creative cognition [21], and autobiographical memory tasks [22]. These results have led to the speculation that an adaptive function of memory retrieval during mind wandering is to facilitate "mental construction of novel episodes to help individuals prepare for the immediate and distant future" [17, p. 262]. 
Further, recent brain imaging studies found that the executive control network (ECN) - a network associated with top-down attention and an external task focus (antithetical to mind wandering) - has also been activated during mind wandering tasking [11, 13]. Similarly, a series of experimental studies have revealed that the DMN increases its activity during goal-directed external cognition, as long as experimental conditions require participants to engage in directed forms of task-unrelated thoughts, such as retrieve episodic, autobiographical information, imagine novel scenes, infer the mental states of other people, self-reflect, etc. [11]. Taken together, these studies suggest that the recruitment of the DMN enables individuals to actively generate mental contents and the ECN helps maintain the goal in order to arrive at the desired action.

In general, the ECN and DMN are thought to act in opposition to each other and the activation of one network typically corresponds to the deactivation of another network [13]. Unlike the DMN, the ECN is responsible for high-level cognitive functions, including the control of attention and top-down processing [23] and is highly active during externally directed attention, such as working memory, relational integration, response inhibition, and task switching [13, 15]. Supporting evidence from neuroimaging studies has shown that the dorsal lateral prefrontal cortex (DLPFC) - a core hub of the ECN-is consistently active during goal-directed tasks [15]. The DLPFC has been widely known as being engaged in divergent thinking [24], metaphor production [25], and creative objects production [21]. These results have been taken as evidence to support the role of the ECN in selfgenerated thought. The activation of both the DMN and ECN has led researchers to suggest that complex cognitive processes, particularly those associated with goal-directed internal activity, involve the dynamic interaction between the DMN and ECN.

Recently, research has identified the third core brain network that potentially modulates the dynamic relationship between the DMN and ECN. However, the literature offers disparate views on the third network involved in the relationship. Some researchers (e.g., [23]) argued that the "salience network", comprised of the anterior insula (AI) and anterior cingulate cortex (ACC), is the third network involved in switching between the default and control networks. The AI has been involved in task-level control, performance monitoring, and focal attention capture [26] and the ACC has been associated with monitoring for competition among potential responses and processes [27]. The close connectivity between the AI and ACC has been observed in task performance involving selfregulatory activities [28]. In healthy subjects, increased activation in the salience network is associated with the self-regulation of internal states [29]. Thus, the salience network forms a core system for the implementation of self-regulation [26] and is thought to act as a mediator between the external and internal worlds [23].

In contrast, other researchers (e.g., [3], [22]) identified the "frontoparietal control network" (FPCN) as the third network and argued that this network plays a crucial role in goal-directed cognition by flexibly coupling with either the default or attention control network. Despite some inconsistency in identifying the precise anatomical boundaries of the FPCN regions, the brain regions identified as the FPCN are overlapping with those associated with the salience network. Prior studies suggest that the FPCN comprises many areas identified as supporting cognitive control, including the ACC, AI, lateral prefrontal cortex, and inferior parietal lobule [30]. The ACC and AI, the brain regions associated with the salience network, are widely associated with cognitive (top-down) control and conflict monitoring. The lateral prefrontal cortex plays a crucial role in supporting many of the complex cognitive operations needed for successful self-regulation.

Drawing on these neuroimaging studies, we develop our conceptual framework as presented in Figure 1. In developing our theoretical framework, we consider the relationship between the large-scale brain networks discussed previously as the foundation for the relationships at the behavioral level.

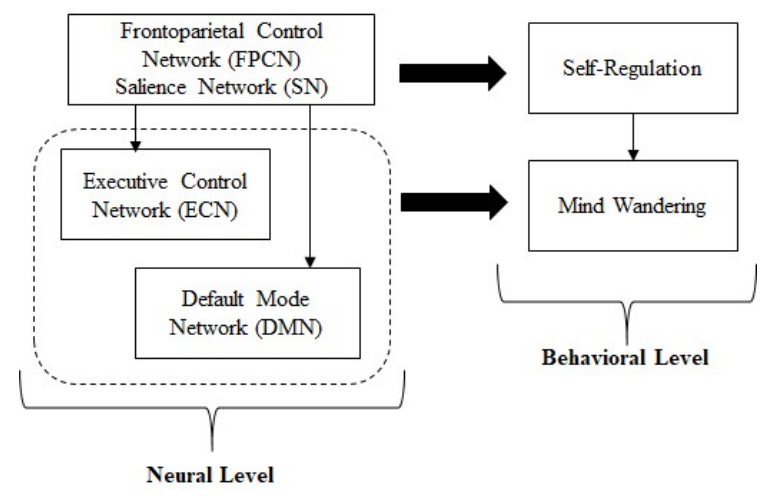

\section{Figure 1. Mapping Brain Functions to the Study Constructs}

The reviewed evidence suggests that (1) the DMN and ECN are involved in representing information that is personally relevant and supports much of the mental activity underlying self-generated thought or mind wandering (e.g., [11]); (3) the FPCN (along with the salience network) plays an important role in regulating and controlling one's thought, behavior, and emotion; thus, is the core hub of self-regulatory activity [31]. 


\section{Research Model and Hypotheses Development}

In presenting our research model below, we are considering the application of IT that supports some tasks (IS tasks) is embedded within the context of human-computer interaction. We specifically focus on the role of mind wandering and self-regulation in influencing cognitive absorption during technology use. Our goal is to test the theoretical framework (Figure 1) at the behavioral level from an IS perspective.

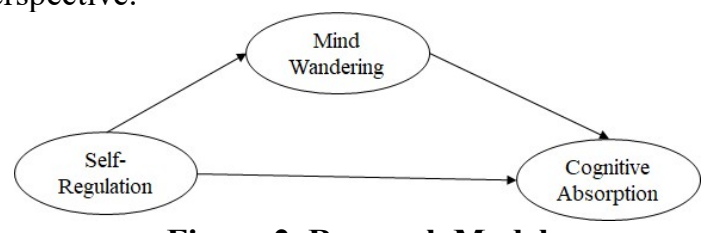

Figure 2. Research Model

\subsection{Self-Regulation and Mind Wandering}

Self-regulation enables people to make plans, choose from alternatives, inhibit unwanted thought, and regulate their behaviors in the presence of conflicts [31]. Specifically, once a goal is established as a reference point for individuals to attain, self-regulation is the means by which individuals evaluate, approach, and attain this goal $[32,33]$. To attain the goal, individuals must regulate their cognitions and behaviors by devoting their effort and attention towards the tasks [33]. Traditionally, the regulation of one's behavior in the pursuit of personal goals has been assumed to happen in a consciously control fashion, and thus, it requires limited executive resources [8; 34]. However, recent evidence has indicated that much of the regulation of our cognition and behavior can occur in a nonconscious fashion by the interplay of situational cues, mental representations of desired stages, and routinized behaviors that can be executed in an efficient yet flexible way [34].

Kanfer and Ackerman proposed three interdependent activities of self-regulation: selfmonitoring, self-evaluation, and self-reaction [8]. Selfmonitoring refers to "the individual's allocation of attention to specific aspects of his or her behavior as well as the consequences of the behavior" [8, p. 662]. Self-monitoring usually occurs in response to internal or external goals. For example, if performance outcomes are considered important, then more attentional resources are allocated to observe those outcomes. Self-evaluation (i.e., performance evaluation) involves "a comparison of current performance with the desired goal state; individuals check their progress against a standard or referent" $[8$, p. 662]. Lastly, self-reaction involves self-satisfaction and perceptions of task-specific capabilities [8].

We hypothesize the effect of self-regulation on mind wandering can be either positive or negative. When engaging in mind wandering activities during performance task is perceived as a distraction, selfregulation will redirect cognitive resources back to the task [35]. However, self-regulatory thought itself can be a distraction. For example, when one is writing code functions, her thought about how others know better about the code, how other's ability is better than hers (i.e., self-evaluation) can increase her tendency to mind wander. Thus, self-regulation can have a positive effect on mind wandering. Another perspective suggests selfregulation helps individuals flexibly switch between tasks and their internal states of mind - to retrieve cognitive elements from memory [36]. To retrieve these cognitive elements, users will disengage from the tasks and likely to enter a state of mind wandering. Thus, self-regulation is likely to have a positive effect on mind wandering.

H1: Self-regulation has a positive effect on mind wandering.

\subsection{Mind Wandering and Cognitive Absorption}

Cognitive absorption refers to a state of deep involvement with technology and captures the holistic experiences with technology, as manifest in (a) total concentration in an activity and (b) the enjoyment which one derives from an activity [37]. Cognitive absorption is defined as a multidimensional construct, consisting of five dimensions: (1) temporal dissociation - the sense of time becomes distorted while engaged in technology; (2) focused immersionthe experience of total engagement where other distractions are ignored; (3) heightened enjoymentthe pleasurable aspects of the interaction; (4) controlthe perception of being in charge of the interaction; and (5) curiosity - the extent the experience of engaging with technology arouses individual's sensory and cognitive curiosity [37]. In a recent study, BurtonJones and Straub considered cognitive absorption as a way to measure users' engagement with the systems during use [38]. In the context of this research, we conceptualize cognitive absorption in a similar way as Burton-Jones and Straub did, to capture a rich experience of system/software usage itself.

We propose the relationship between mind wandering and cognitive absorption is curve-linear ( $\mathrm{U}$ shape). Initially, mind wandering is expected to have a negative effect on cognitive absorption. Mind wandering can impair the encoding of information and take away limited cognitive resources, leading to a 
failure to engage in the main task [39]. However, mind wandering can also be used as a mental break when one is trying to solve a problem. A number of experiments have shown that people who are stumped in solving certain kinds of problems are subsequently much more likely to solve the problem if they think about something else (engaged in mind wandering) [40]. We argue that this relationship is curve-linear, mind wandering can have a negative effect on cognitive absorption to a point it turns positive and helps an individual engage in an activity.

H2: There is a U shape relationship between mind wandering and cognitive absorption.

\subsection{Self-Regulation and Cognitive Absorption}

Resource allocation theory has been widely tested in the learning and training contexts (e.g., [41]). The findings reveal that the effectiveness of self-regulation strategies determines learning outcomes [8, 41]. Since the most demanding stage of technology use is during the early phase of learning [41], self-regulatory activities will serve to increase resource allocations to engage in the relevant tasks. Thus, we hypothesize self-regulation has a positive effect on cognitive absorption.

H3: Self-regulation has a positive effect on cognitive absorption

\section{Research Methods}

\subsection{Study Context and Sample}

The research approach taken to empirically test the research model was a field study using an online survey methodology for data collection. We collected data from student subjects enrolled in a large public university in the United States. Students enrolled in the introductory IS course in the college of business were surveyed as an exchange for course credit. This course is mandatory to all students who major in information systems. As a part of their assignment, students were instructed to complete a self-pace tutorial on Microsoft Access, specifically on exchanging data between Access and other applications. After they completed the tutorial, they had to solve a business case study from the handbook that required them to accurately export and display business data into appropriate formats. To minimize the recall and memory bias, participants were instructed to fill out a survey and report their learning experience on Microsoft Access immediately after they completed their assignment. The use of questionnaire or survey is suitable for this study because it has a higher generalizability and greater external reliability as they are based on the actual users' experience [42]. In total, a sample of 323 valid responses was collected. Of the 323 respondents, 53 percent were males and over 65 percent were in the 21 to 25 age range. Over 85 percent of the respondents were English native speakers.

\subsection{Operational of Research Variables}

All research variables were measured using multiitem seven-point Likert scales. Scales for mind wandering were adopted from Sarason et al. [43]. Examples of mind wandering items are: "[When I use MS. Access to complete my task], I thought about members of my family"; "..., I thought about friends"; and "..., I thoughts about personal worries." Selfregulation was conceptualized as a multidimensional, reflectively measured construct and the scales were adapted from Kanfer and Ackerman [8] and Grafill and Compeau [41]. Examples of self-regulation items are "I thought about how others would have known better about MS. Access"; "I monitored how well I was learning MS. Access"; and "I thought about how much my knowledge about MS. Access has improved". The scales to measure cognitive absorption were adapted from Agarwal and Karahanna [37]. Consistent with Agarwal and Karahanna, we conceptualize cognitive absorption as a multidimensional, reflectively measured construct [37].

\section{Data Analysis and Results}

\subsection{Measurement Model}

The measurement model was tested using the component-based partial least square (PLS) approach. PLS is appropriate for this study because it focuses on predicting key target constructs and is well suited for exploratory models and theory development [44]. The Smart-PLS software package (version 3.2) was used for the estimations. Since the measures for selfregulation and cognitive absorption consisted of second-order factors and Smart-PLS does not directly permit the representation of second-order latent constructs, we used the sequential latent variable score method or two-stage approach [37]. We first estimated the construct scores of the first-order constructs in the first-stage model without the second-order construct present, and subsequently used these factor scores as indicators for the second order latent variables in the second-stage model [37].

Descriptive statistics for the key constructs are shown in Table 1. The psychometric properties of the scales were measured by observing internal 
consistency scores, convergent, and discriminant validity. Internal consistency scores of all variables, calculated by the composite reliability scores, are considered acceptable since they exceed .70, signifying tolerable reliability (see Table 1). Convergent and discriminant validity is inferred when (1) the indicators load higher on their hypothesized factor than on other factors, and (2) the square root of each construct's average variance extracted (AVE) is larger than the inter-construct correlations [45]. The Confirmatory Factor Analysis results demonstrate that all items loaded well on their respective factors, which are much higher than all cross loadings.

Table 1. Descriptive Statistics

\begin{tabular}{lcccc}
\hline \multicolumn{1}{c}{ Construct } & Mean & SD & CR & AVE \\
\hline Mind Wandering & 3.49 & 1.42 & .95 & .68 \\
SL (Self-Monitoring) & 3.97 & 1.36 & .94 & .80 \\
SL (Self-Evaluation) & 3.41 & 1.65 & .96 & .89 \\
SL (Self-Reaction) & 3.89 & 1.35 & .90 & .70 \\
CA (Temp. Diss.) & 4.39 & 1.29 & .89 & .68 \\
CA (Focused Immersion) & 4.49 & 1.18 & .92 & .73 \\
CA (H. Enjoyment) & 3.80 & 1.45 & .95 & .88 \\
CA (Control) & 4.39 & 1.21 & .88 & .73 \\
CA (Curiosity) & 3.76 & 1.54 & .97 & .94 \\
\hline Notes: SL = Self-Regulation; CA $=$ Cognitive Absorption; CR & CA \\
Composite Reliability; AVE & $=$ Average Variance Extracted. \\
Dependent Variable: CA; Independent Variables: Mind Wandering \\
and SL.
\end{tabular}

Furthermore, as can be in Table 2, the square root of all AVEs are above .80, which are much higher than all the inter-correlations. Taken together, these tests suggest that all measures have adequate convergent and discriminant validity. Common method bias was assessed using Harman's one factor test [46]. Each factor construct explains roughly equal variance, indicating that common method bias is not a major issue in our data. We also ran a common method variance test with a common latent factor in AMOS [46]. The estimated amount of method bias was only 1.2 percent, indicating our data do not suffer from high common method variance.

Table 2. Correlation Matrix

\begin{tabular}{|c|c|c|c|c|c|c|c|c|c|c|}
\hline & Construct & 1 & 2 & 3 & 4 & 5 & 6 & 7 & 8 & 9 \\
\hline 1 & Mind Wandering & .83 & & & & & & & & \\
\hline 2 & $\begin{array}{l}\text { SL (Self- } \\
\text { Monitoring) }\end{array}$ & & .90 & & & & & & & \\
\hline 3 & $\begin{array}{l}\text { SL (Self- } \\
\text { Evaluation) }\end{array}$ & $.43^{* *}$ & $.55^{* * *}$ & .94 & & & & & & \\
\hline 4 & SL (Self-Reaction) & .06 & $.54^{* *}$ & $.30^{* *}$ & .84 & & & & & \\
\hline 5 & CA (Temp. Diss.) & $.18^{* * *}$ & $.20^{* *}$ & $.24 * *$ & $.13^{*}$ & .82 & & & & \\
\hline 6 & $\begin{array}{l}\text { CA (Focused } \\
\text { Immersion) }\end{array}$ & -.07 & $.33^{* *}$ & $.13^{*}$ & $.39 * *$ & $.26^{* *}$ & .86 & & & \\
\hline 7 & $\mathrm{CA}$ (H. Enjoyment) & .08 & $.36^{* *}$ & $.27^{* *}$ & $.43^{* *}$ & $.30^{* *}$ & $.44^{* *}$ & .94 & & \\
\hline 8 & $\mathrm{CA}$ (Control) & $-.13^{*}$ & $.28^{* *}$ & .01 & $.26^{* *}$ & $.14^{* * *}$ & $.51^{* *}$ & $.51^{* *}$ & .89 & \\
\hline 9 & $\mathrm{CA}$ (Curiosity) & $.12^{*}$ & $.33 * *$ & $.24 * *$ & $.39 * *$ & $.25 * *$ & $.39 * *$ & $.76 * *$ & $.47^{* *}$ & .97 \\
\hline
\end{tabular}


environment [47]. However, simply engaging in mind wandering is insufficient for reaching cognitive absorption. Only when mind wandering is up to a certain point, cognitive absorption is achieved. When a problem is set aside, a goal representation remains active for extended time periods [48]. The active goal representation during the experience of mind wandering would increase cognitive absorption as users are creating solution-relevant paths. This could also explain the dynamic interaction between the DMN and ECN during goal-directed activity.

The findings also suggest the importance role of self-regulation. In order to remain constantly engaged and motivated, a user needs to have a clear sense of how well his or her skills are meeting the demands of the environment [47]. Having the ability to make an adjustment needed to maintain performance will help a user achieve the state of cognitive absorption while at the same time, weight the costs of engaging in mind wandering against its benefits. For example, if the selfregulatory system notices one has sufficient cognitive resources to engage in mind wandering and such activity will facilitate learning, then resources will be allocated to mind wandering experience while the relevant goal is kept active, although the process is not always conscious.

\subsection{Theoretical Implications}

We view our current study as a starting point for future investigating, specifically on the potential of advancing the attentional processes involved in the interaction between users and technology. Given research in mind wandering itself is relatively new in the reference discipline, the assumptions underlying our theoretical model are almost certainly incomplete. Thus, it provides research opportunities for the IS community to explore the roles of internal cognition in IS research. First and foremost, we draw our research model primarily from functional imaging studies to theoretically derive the relevant constructs. By developing the theory from the reference discipline, we extend our knowledge on how to translate the existing neurological processes into behavioral processes that have not been identified in the previous studies.

Second, our findings suggest there is a U-Shape relationship between mind wandering and cognitive absorption. It has been acknowledged that focused attention is should precede cognitive absorption [37]. In contrast to this argument, we found mind wandering could have a positive effect on cognitive absorption. Engaging in mind wandering during cognitive tasks (e.g., technology use) may help users enter the incubation period in which the DMN and ECN mutually contribute to associative processes [6]. This incubation period may lead to increased cognitive absorption. With this new insight, our study potentially speaks to the unique value that mind wandering may offer in the technology use process.

Third, the findings of this study also shed light and have implications on the roles of self-regulation in technology use. Although ongoing investigation is needed to examine the importance of self-regulation in managing the state of mind wandering (e.g., ensuring users have enough cognitive resources to execute the main task), the findings of our current study demonstrate that self-regulation positively contributes to mind wandering. Due to its critical roles in managing internal-directed and external-directed cognition, we suggest researchers not to omit the effect of self-regulation when they study attentional mechanisms during technology use. The possible omission of the effect of self-regulation may distort findings and alter the study's interpretation.

Further, although prior IS research has explored the necessity of external attention to guide behavior (e.g., users' perceptions toward technology determine their attitude and behaviors), research on internally directed attention has been rare, despite the fact that people claim to be studying "the mental representation to produce the problem solving" [49, p. 66]. By introducing and integration mind wandering into technology use and showing it has a curve-linear relationship with cognitive absorption, this study integrates internally directed cognition to understand how users think and process information during technology use. The findings reveal that when mind wandering reaches a certain point, it may increase accessibility to the problem at hand. This increased accessibility may serve as a functional role by enabling novel ideas to spring in mind [50], yielding positive affect that marks the realization of successful relevant processing [47].

We argue that further progress in our understanding of human-computer interaction would be impossible without considering internally directed cognition, which entails some of the biggest challenges for researchers to resolve and yet holds some of the biggest promises for the advance of our theory development [11]. While we borrow insight, theory, and findings from the reference disciplines, our intention is to understand IS phenomena where attentional processes are involved. Although we are far from a full understanding of these attentional mechanisms, what we can infer from our current findings points primarily to the potential benefits of mind wandering in our technological environment.

\subsection{Practical Implications}


This paper makes several practical implications by demonstrating the relationship between self-regulation, mind wandering, and cognitive absorption. The application of internally directed cognition has not been systematically introduced to the education as well as business contexts. This study findings suggest that to increase one's cognitive absorption during technology use, educators or practitioners should not prevent users from engaging in mind wandering. Although mind wandering seems to reduce cognitive absorption at the early stage of use, this process can be reversed if mind wandering is activated during goaldirected behaviors. The activation of DMN and ECN is the evidence for the activation of mind wandering during goal-directed behaviors.

For managers, this study implies that optimizing "regulated mind wandering" can help facilitate effective technology use. Managers should allow users to flexibly regulate their thoughts around the main task while also encourage them to freely engage in mind wandering when mental load is high. Failure to maintain control over self-regulation will lead to undesirable consequences for technology users. Several interventions for boosting self-regulation have been suggested in the cognitive psychology literature [51], and some of these could be used to train IT workers as well. For example, organizations can employ a project management intervention program designed to assist IT workers with implementing a regular project schedule by tracking their activity and productivity and provide feedbacks on a daily basis. Such interventions may help IT workers to attain their goals that may lead to a systematic activation of the $\mathrm{DMN}$, ECN, or both, depending on the tasks constraints. Other interventions program may increase self-regulation by unconsciously activating goals that can lead directly to motivated behaviors (i.e., priming technique).

\subsection{Limitations}

Several limitations of the current study should be taken into account when interpreting its findings. First, the theoretical model developed and tested in this study is primarily derived from the brain imaging studies that should be subjected to further testing and validation processes. Support for the proposed model should be tested using a strategy of triangulation, whereby selfreport, behavioral measures, and neuro imaging studies are used together to make inferences about the phenomena under study. Second, our sample was drawn from university students. Although using the university students was appropriate, future research can take this investigation further by drawing research subjects from a more diverse population. Third, the context of the study is a concern. As this study exclusively focuses on users interaction when they use MS. Access, our results may or may not be generalize to other contexts and other types of software. Further research could and should empirically test the model in other contexts using different types of technology.

\section{Conclusion and Future Research}

This paper provides an additional evidence that mind wandering can be beneficial for technology users - the relationship between mind wandering and cognitive absorption is curvilinear. As mind wandering increases, cognitive absorption decreases to a certain point, after which, cognitive absorption increases as mind wandering increases. The findings also reveal the importance of self-regulation in facilitating cognitive absorption and mind wandering. Mind wandering can be regarded as intrinsically desirable and valued when it is used during goal-directed behaviors.

We need to acknowledge that mind wandering is relatively new as a subject of research, particularly in the IS discipline, and thus through this study, we suggest several avenues for future research to investigate the antecedents and/or consequences of mind wandering in IS research. For example, does mind wandering influence software development processes? In what situations are mind wandering experiences more beneficial? How do different types of system designs facilitate mind wandering or focused attention among developers as well as users? How can a balance between focused attention and mind wandering be achieved to maximize performance with using a system? Further, the theoretical model and findings reported in this study provide a more complete explanation of the attentional mechanisms involved in internally directed attention, which adds to the base of knowledge from which future research can draw upon and continue making progress toward a better understanding of how users process their thought during their interaction with technology.

\section{References}

[1] Burton-Jones, A., and Grange, C. "From Use to Effective Use?: A Representation Theory Perspective," Information System Research, 24(3), 2013, pp. 623-658.

[2] Smallwood, J., and Schooler, J. W. "The Restless Mind," Psychological Bulletin, 132(6), 2006, pp. 946-958.

[3] Benedek, M., Jauk,E., Beaty, R. E., Fink, A., Koschutnig, K., and Neubauer, A. C. "Brain Mechanisms Associated with Internally Directed Attention and Self-Generated Thought," Scientific Reports, 6(22959), 2016, pp. 1-8. 
[4] McVay, J. C., and Kane, M. J. "Conducting the Train of Thought: Working Memory Capacity, Goal Neglect, and Mind Wandering in an Executive-Control Task," Journal of Experimental Psychology. Learning, Memory, and Cognition, 35(1), 2009, pp. 196-204.

[5] Smallwood, J., Beach, E., Schooler, J. W., and Handy, T. C. "Going AWOL in the Brain: Mind Wandering Reduces Cortical Analysis of External Events," Journal of Cognitive Neuroscience, 20(3), 2008, pp. 458-469.

[6] Baird, B., Smallwood, J., and Schooler, J. W. "Back to the Future: Autobiographical Planning and the Functionality of Mind-Wandering," Consciousness and Cognition, 20(4), 2011, pp. 1604-1611.

[7] Christoff, K., Gordon, A., Smith, R., and Vancouver, B. C. "The role of spontaneous thought in human cognition. In Neuroscience of Decision Making," O. Vartanian, D. R. Mardel (eds.), 2011, pp. 259-284, New York, NY: Psychology Press.

[8] Kanfer, R., and Ackerman, P. L. Motivation and Cognitive Abilities: An Integrative/Aptitude-Treatment Interaction Approach to Skill Acquisition," Journal of Applied Psychology, 74(4), 1989, pp. 657-690.

[9] Andrews-Hanna, J. R., Kaiser, R. H., Turner, A. E. J., Reineberg, A. E., Godinez, D., Dimidjian, S., and Banich, M. T. "A Penny for Your Thoughts: Dimensions of Selfgenerated Thought Content and Relationships with Individual Differences in Emotional Wellbeing," Frontiers in Psychology, 4(November), 2013, pp. 1-13.

[10] Killingsworth, M. A., and Gilbert, D. T. "A Wandering Mind is an Unhappy Mind," Science, 330(6006), 2010, pp. 932-932.

[11] Andrews-Hanna, J. R., Smallwood, J., and Spreng, R. N. "The Default Network and Self-generated Thought: Component Processes, Dynamic Control, and Clinical Relevance," Annals of the New York Academy of Sciences, 1316(1), 2014, pp. 29-52.

[12] Allen, M., Smallwood, J., Christensen, J., Gramm, D., Rasmussen, B., Jensen, C. G., ... Lutz, A. "The Balanced Mind: The Variability of Task-Unrelated Thoughts Predicts Error Monitoring," Frontiers in Human Neuroscience, 7, 2013, pp. 1-15.

[13] Christoff, K., Gordon, A. M., Smallwood, J., Smith, R., and Schooler, J. W. "Experience Sampling During fMRI Reveals Default Network and Executive System Contributions to Mind Wandering," Proceedings of the National Academy of Sciences, 106(21), 2009, pp. 87198724.

[14] Christoff, K., Ream, J. M., and Gabrieli, J. D. "Neural Basis of Spontaneous Thought Processes," Cortex, 40(4), 2004, pp. 623-630.
[15] Beaty, R. E., Benedek, M., Kaufman, B. S., and Silvia, P. J. "Default and Executive Network Coupling Supports Creative Idea Production. Scientific Reports, 5(10964), 2015, pp. 1-14.

[16] Smallwood, J., and Schooler, J. W. "The Science of Mind Wandering: Empirically Navigating the Stream of Consciousness," Annual Review of Psychology, 66(1), 2015, pp. 487-518.

[17] Andrews-hanna, J. R. "The Brain's Default Network and Its Adaptive Role in Internal Mentation," The Neuroscientist, 18(3), 2012, pp. 251-270.

[18] Brewer, J. A, Garrison, K. a, and Whitfield-Gabrieli, S. "What about the "Self" is Processed in the Posterior Cingulate Cortex?," Frontiers in Human Neuroscience, 7(October), 2013, pp. 1-7.

[19] Northoff, G., Heinzel, A., de Greck, M., Bermpohl, F., Dobrowolny, H., and Panksepp, J. "Self-Referential Processing in Our Brain: A Meta-Analysis of Imaging Studies on the Self," NeuroImage, 31(1), 2006, pp. 440-457.

[20] Buckner, R. L., and Carroll, D. C. "Self-Projection and the Brain," Trends in Cognitive Sciences, 11(2), 2007, pp. 49-57.

[21] Ellamil, M., Dobson, C., Beeman, M., and Christoff, K. "Evaluative and Generative Modes of Thought During the Creative Process," Neuroimage, 59(2), 2012, pp. 1783-1794.

[22] Spreng, R. N., and Grady, C. L. "Patterns of Brain Activity Supporting Autobiographical Memory, Prospection, and Theory of Mind, and Their Relationship to the Default Mode Network," Journal of Cognitive Neuroscience, 22, 2010, pp. 1112-1123.

[23] Bressler, S. L., and Menon, V. "Large-scale Brain Networks in Cognition: Emerging Methods and Principles," Trends in Cognitive Sciences, 14(6), 2010, pp. 277-290.

[24] Seger, C. A., Desmond, J. E., Glover, G. H., and Gabrieli, J. D. "Functional Magnetic Resonance Imaging Evidence for Right-hemisphere Involvement in Processing Unusual Semantic Relationships," Neuropsychology,14(3), 2000, pp. 361-369.

[25] Benedek, M., Jauk, E., Sommer, M., Arendasy, M., and Neubauer, A. C. "Intelligence, Creativity, and Cognitive Control: The Common and Differential Involvement of Executive Functions in Intelligence and Creativity," Intelligence, 46(1), 2014, pp. 73-83.

[26] Nelson, S. M., Dosenbach, N. U. F., Cohen, A. L., Wheeler, M. E., Schlaggar, B. L., and Petersen, S. E. "Role of the Anterior Insula in Task-level Control and Focal Attention," Brain Structure and Function, 2010, pp. 1-12.

[27] Kounios, J., Frymiare, J.L., Bowden, E.M., Fleck, J.I., Subramaniam, K., Parrish, T.B., et al. "The Prepared Mind: 
Neural Activity Prior to Problem Presentation Predicts Subsequent Solution by Sudden Insight," Psychological Science 17, 2006, pp. 882-890.

[28] Posner, M., and Rothbart, M. K. "Toward a Physical Basis of Attention and Self-regulation," Psychology Life Review, 6(2), 2009, pp. 103-120.

[29] Bonnelle, V., Ham, T. E., Leech, R., Kinnunen, K. M., Mehta, M. A., Greenwood, R. J., and Sharp, D. J. "Salience Network Integrity Predicts Default Mode Network Function After Traumatic Brain Injury," Proceedings of the National Academy of Sciences, 109(12), 2012, pp. 4690-4695.

[30] Spreng, R. N., Sepulcre, J., Turner, G. R., Stevens, W. D., and Schacter, D. L. "Intrinsic Architecture Underlying the Relations Among the Default, Dorsal Attention, and Frontoparietal Control Networks of the Human Brain," Journal of Cognitive Neuroscience, 25(1), 2013, pp. 74-86.

[31] Kelley, W. M., Wagner, D. D., and Heatherton, T. F. "In Search of a Human Self-regulation System," Annual Review of Neuroscience, 38(1), 2015, pp. 389-411.

[32] Locke, E., and Latham,G. theory of goal setting and task performance. 1990. Englewood Cliffs, NJ: Prentice-Hall, Inc.

[33] Brusso, R. C., and Orvis, K. A. "The Impeding Role of Initial Unrealistic Goal-setting on Videogame-based Training Performance: Identifying Underpinning Processes and a Solution," Computers in Human Behavior, 29(4), 2013, pp. 1686-1694.

[34] Papies, E. K., and Aarts, H. "Nonconscious SelfRegulation, or the Automatic Pilot of Human Behavior," In Handbook of Self-Regulation (2nd ed.), K. D. Vohs \& R. F. Baumeister (Eds.), 2011, pp. 125-142, New York, NY: The Guilford Press.

[35] Dimitrova, N. G., Dyck, C., Hooft, E. A., and Groenewegen, P. "Don't Fuss, Focus: The Mediating Effect of On-task Thoughts on the Relationship Between Error Approach Instructions and Task Performance," Applied Psychology, 64(3), 2015, pp. 599-624.

[36] Sacramento, C. A., Fay, D., and West, M. A. "Workplace Duties or Opportunities?? Challenge Stressors, Regulatory Focus, and Creativity," Organizational Behavior and Human Decision Processes, 121(2), 2013, pp. 141-157.

[37] Agarwal, R., and Karahanna, E. "Time Flies When You're Having Fun: Cognitive Absorption and Beliefs About Information Technology Usage," MIS Quarterly, 24(4), 2000, pp. 665-694.

[38] Burton-Jones, A., and Straub Jr, D. W. "Reconceptualizing System Usage: An Approach and Empirical Test," Information Systems Research, 17(3), 2006, pp. 228-246.
[39] Smallwood, J., Fishman, D. J., and Schooler, J. W. "Counting the Cost of an Absent Mind: Mind Wandering as An Underrecognized Influence of Educational Performance," Psychological Bulletin Review, 14, 2007, pp. 230-236.

[40] Gable, S. L., Hopper, E. A., and Schooler, J. W. When the Muses Strike: Creative Ideas of Physicists and Writers Routinely Occur During Mind Wandering," Psychological science, 30(3), 2019, pp. 396-404.

[41] Gravill, J., and Compeau, D. "Self-regulated Learning Strategies and Software Training," Information \& Management, 45(5), 2008, pp. 288-296.

[42] Churchill, G. A. J., and Iacobucci, D. Marketing Research: Methodological Foundations, 9th ed., 2005. Thomson Learning. Versailles, KY.

[43] Sarason, I.G., Sarason, B.R., Keefe, D.E., Hayes, B.E., and Shearin, E.N. "Cognitive Interference: Situational Determinants and Traitlike Characteristics," Journal of Personality and Social Psychology, 51, 1986, pp. 215-226.

[44] Hair, J. F., Ringle, C. M., and Sarstedt, M. "PLS-SEM: Indeed a Silver Bullet," Journal of Marketing Theory and Practice, 19(2), 2011, pp. 139-152.

[45] Chin, W. "The Partial Least Squares Approach to Structural Equation Modeling," In Modern Methods for Business Research. G. A. Marcoulides (ed.), 1998. Lawrence Erlbaum Associates. Mahwah, NJ.

[46] Podsakoff, P. M., MacKenzie, S. B., Lee, J.-Y., and Podsakoff, N. P. "Common Method Biases in Behavioral Research: A Critical Review of The Literature and Recommended Remedies," Journal of Applied Psychology, 88(5), 2003, pp. 879-903.

[47] Vervaeke, J., Ferraro, L., and Herrera-Bennett, A. "Flow as spontaneous thought: Insight and implicit learning," In Oxford Handbooks Online, K. Christoff and K. C. R. Fox (Eds.), 2018, pp. 1-28, Oxford, UK: Oxford University Press.

[48] Gilhooly, K. J. 2016. "Incubation and intuition in creative problem solving," Frontiers in Psychology (July), 2016, pp. 1-9.

[49] Vessey, I., and Galletta, D. "Cognitive Fit: An Empirical Study of Information Acquisition," Information Systems Research, 2(1), 1991, pp. 63-84.

[50] Gable, S. L., Hopper, E. A., and Schooler, J. W. "When the Muses Strike: Creative Ideas of Physicists and Writers Routinely Occur During Mind Wandering," Psychological Science, 30(3), 2019, pp. 396-404.

[51] Bauer, I. M., and Baumeister, R. F. "Self-Regulation Strength," In Handbook of Self-Regulation (2nd ed.). K. D. Vohs \& R. F. Baumeister (Eds.), 2011, pp. 64-82, New York, NY: The Guilford Press. 